\title{
Reliability and validity of the Polish version of the Achilles tendon Total Rupture Score
}

\author{
Paweł Bąkowski ${ }^{1} \cdot$ Szymon Rubczak $^{1} \cdot$ Maria Wolff-Stefaniak $^{1}$ • \\ Monika Grygorowicz ${ }^{2} \cdot$ Tomasz Piontek $^{1,3}$
}

Received: 6 May 2017 / Accepted: 23 October 2017 / Published online: 1 November 2017

(c) The Author(s) 2017. This article is an open access publication

\begin{abstract}
Purpose The aim of this study was to perform the translation and cross-cultural adaptation of the Achilles tendon Total Rupture Score (ATRS) into Polish version, and to evaluate its reliability and validity.

Methods The ATRS was translated into Polish language according to the Beaton recommendations. A total number of 71 patients previously treated surgically (from 2011 to 2015), due to the Achilles tendon rupture, were enrolled in this study. ATRS-Polish was performed twice within a period of 5-10 days. To evaluate test-retest reliability, intrarater coefficient (ICC) was calculated. Construct validity was determined by the Spearman's rank coefficient correlation
\end{abstract}

Electronic supplementary material The online version of this article (doi:10.1007/s00167-017-4764-7) contains supplementary material, which is available to authorized users.

Paweł Bąkowski

pawel.bakowski@rehasport.pl

Szymon Rubczak

szymon.rubczak@rehasport.pl

Maria Wolff-Stefaniak

maria.wolff-stefaniak@ rehasport.pl

Monika Grygorowicz

monika.grygorowicz@rehasport.pl

Tomasz Piontek

tomasz.piontek@rehasport.pl

1 Orthopedic Department, Rehasport Clinic, Górecka 30, 60-201 Poznan, Poland

2 Research and Development Department, Rehasport Clinic, Poznan, Poland

3 Department of Spine Disorders and Pediatric Orthopedics, University of Medical Sciences Poznań, Poznan, Poland between the ATRS-Polish and a Polish version of EQ-5D-5L questionnaire.

Results Test-retest reliability was found to be excellent (ICC 0.9). The mean and standard deviation of the first and second assessment amounted $87.4 \pm 14.0$ and $88.4 \pm 13.2$, respectively. Construct validity analysis showed a strong correlation between the ATRS and the EQ-5D-5L score $(r=-0.69$. $)$ and moderate correlation between ATRS and actual comfort $(r=0.47)$.

Conclusions and perspectives Polish version of the Achilles tendon Total Rupture Score was found to be reliable and valid.

Level of evidence Level II.

Keywords Achilles tendon rupture - ATRS - Crosscultural $\cdot$ Validity $\cdot$ Reliability $\cdot$ Polish

\section{Introduction}

The Achilles tendon is the largest and strongest tendon in the human body [19]. Despite this fact, the Achilles tendon is considered the most commonly injured tendon in the lower extremity [1]. Current estimates show an incidence of about 5-10 ruptures (range $8.3-24$ ) per 100,000 population [12, 13, 16, 22, 24]. The impact of Achilles tendon ruptures can be deeply felt across many aspects of patients' lives. Therefore, an early diagnosis as well as choosing the proper treatment and rehabilitation programs is of crucial importance.

Up to date, the choice of Achilles tendon rupture treatment method is still ambiguous. Despite a vast array of reports, none of the published studies clearly demonstrate the definitive superiority of one method over another (as reviewed in [17]). In 2010, the American Academy of Orthopaedic Surgeons (AAOS) released 16 recommendations as 
clinical practice guidelines, based on a series of systematic reviews of published studies on the diagnosis and treatment of acute Achilles tendon rupture [7]. None of these was graded as strong recommendations which could be potentially used routinely [23]. Therefore, there is a need for creating an appropriate tool which aims at proper evaluation and reliable comparison of Achilles tendon ruptures treatment methods. Such evaluation of the treatment outcome, especially patient function, is of foreground importance, that is why patient-relevant instruments are commonly used.

The only patient-reported outcome measure (PROM) for Achilles tendon ruptures is the Achilles tendon Total Rupture Score (ATRS). It has been developed in 2007 in Sweden [19]. It consists of ten questions concerning current symptoms and physical activity during Achilles tendon rupture. As an outcome of this experimental setup, a onefactor score reflecting the desired dimension of symptoms and physical activity was achieved. This score comprised 10 items, 11-grade Likert scale each. Till now, ATRS has been successfully used in multiple patient groups, e.g. in the UK population with sustained and isolated acute Achilles tendon rupture [15]. Furthermore, recent studies demonstrated visible improvements in the ATRS during the first year following the surgery [4]. However, the successful employment of the ATRS strictly depends on the patients' ability to read and to understand the language used in the questionnaire. Moreover, one has to take into account also the local culture of examined population. The incompatibilities of sociocultural and economic conditions among different countries make the regular translation insufficient for proper estimation of the ATR score. For these reasons, the ATRS scale has been already translated and culturally adapted for English [5], Danish [9], Italian [26], Turkish [14], Portuguese [27], Dutch [9, 21] Persian [2] and recently also Chinese [8] and Norvegian [18]. Up to date, there is no Polish version of ATRS available, for that authors decided to translate and adapt this score into Polish.

The aim of this study was to perform the cross-cultural adaptation of the ATRS into Polish language and to evaluate its reliability and validity in a sample of Polish respondents.

\section{Materials and methods}

\section{ATRS translation and cross-cultural adaptation}

The ATRS was translated to Polish language according to the Beaton recommendations [3]. The whole translation procedure consisted of five following steps (Fig. 1):

Step 1 Two native Polish speakers translated ATRS from English into Polish language. One translator was a non-medical, second was an orthopaedic surgeon. Both translations

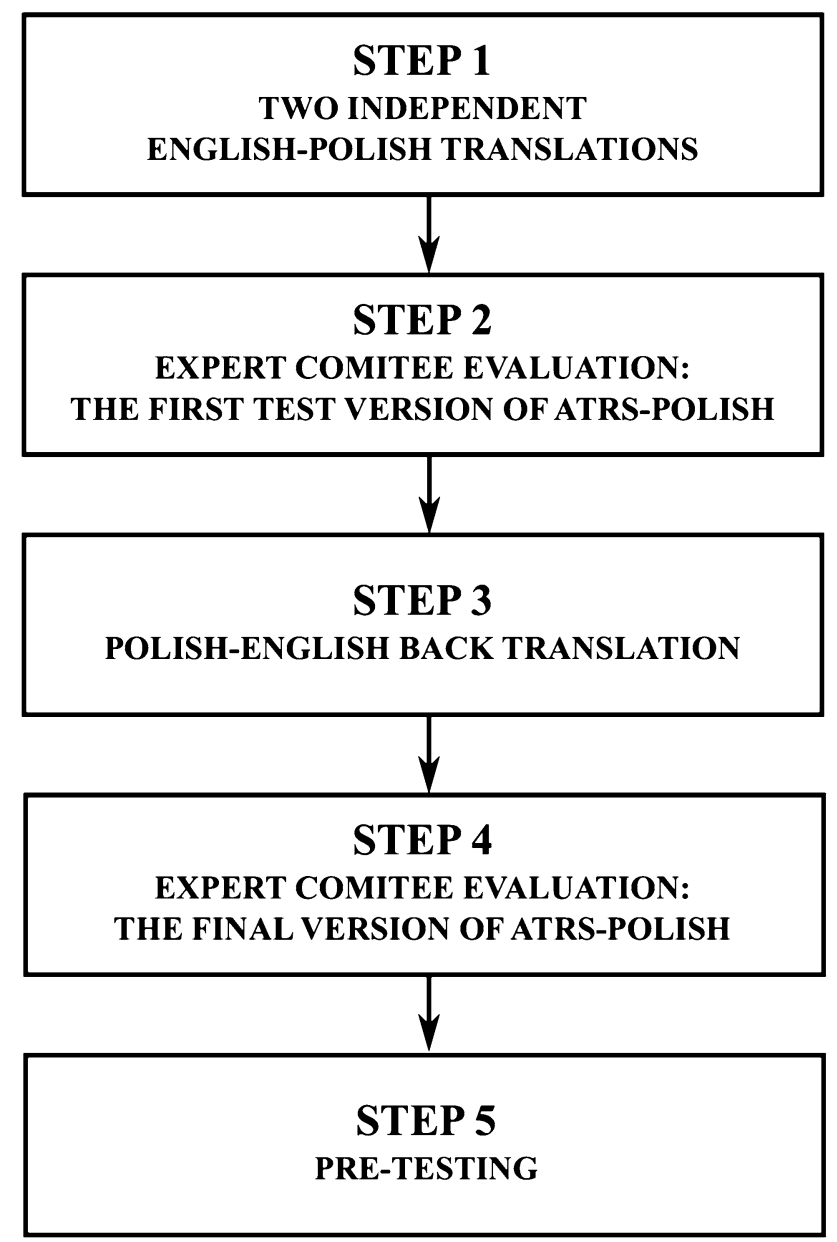

Fig. 1 Flow chart of translation and cross-cultural adaptation of the Achilles tendon Total Rupture Score (ATRS) to Polish language

were performed independently. Only one translator had a prior knowledge of the questionnaire content.

Step 2 Two distinct versions of the translated ATRS were presented to the expert committee consisting of four doctors and one physiotherapist with an expertise in Achilles tendon rupture treatment. The aim of such evaluation was to estimate the accuracy, fluency and style of translated scores as well as to discuss and highlight any possible errors or inconsistencies in translations. As an outcome, the first test version of Polish ATRS was established.

Step 3 Back translation of the Polish ATRS to English language was performed by native English translator. The translator was unaware of the purpose of the present study and had no access to the original English version. This new translation into English allowed for identification of possible translation errors or grammatical inconsistencies, and was compared to the original version.

Step 4 Expert committee reviewed and evaluated all versions of translated ATRS questionnaires. The aim of this step was to estimate the compatibility between all versions 
of test Polish ATRS translations. As an outcome, the final ATRS-Polish version was established and approved.

Step 5 Pre-testing. ATRS-Polish was distributed among ten patients with ankle pain and among ten asymptomatic patients. None of the patients had difficulties in answering the questions. The feedback from the patients revealed the questions were clear and understandable.

\section{ATRS-polish validity}

ATRS-polish questionnaire was compared to the Polish EQ5D-5L test, previously adapted by Golicki et al. [10]. The EQ-5D-5L is a quality of life questionnaire which is widely used for clinical assessment. It consists of five questions regarding the mobility, self-care, usual activities, pain/discomfort and anxiety/depression. Each question comprises five levels (from $1=$ no problem, to $5=$ extreme problem). The less points EQ-5D-5L score gets, the better outcome it describes. Additionally, one question is related to the actual comfort (range from 0 to 100 points).

To assess convergent/divergent validity of ATRS-Polish, Spearman's correlation coefficients and their 95\% confidence intervals were calculated between ATRS-Polish and EQ-5D-5L as well as between ATRS-Polish and actual comfort. Spearman's correlation coefficients were classified as follows: strong for values $>0.5$; moderate for values between 0.35 and 0.5 ; weak correlation for values $<0.35$ [11].

\section{ATRS-polish reliability}

Test-retest reliability of ATRS-Polish was performed within a period of 5-10 days after performing the first test. No treatment was provided during that period to minimize the changes in patient's clinical status. To assess consistency and agreement of test-retest reliability intraclass correlation coefficient (ICC) was used.

To evaluate internal consistency, which refers to homogeneity of all answers within a questionnaire, Cronbach coefficient alpha was used.

\section{Floor and ceiling effect}

Floor and ceiling effect were also assessed, which refer to proportion of the respondents with the maximum (100 points) or minimum (0 points) scores. It is present when more than $30 \%$ of the respondents reach the maximum or minimum scores [14].

Statistical significance was accepted at $p<0.05$. The calculations were performed using the PQStat 1.6.2 environment.

\section{Patients and ethics approval}

A total number of 71 patients who presented Achilles tendon rupture and were treated in Poznań from 2011 to 2015 was enrolled in this study (Table 1). Patients were treated either in Rehasport Clinic in Poznań (49 cases) or in Poznań Regional Hospital (22 cases). 44 of them were treated with a percutaneous suturing, 17 patients by open suturing, in 1 case turn-down-flap suturing was performed and 9 patients received Achilles tendon reconstruction with semitendinosus and gracilis tendon grafts [20]. Left Achilles tendon was treated in 41 cases, in 30 cases right Achilles tendon was ruptured. A study was conducted 6-64 months after surgery (with a mean value of $30.0+/-13.0$ months). 68 patients were eligible for inclusion in a reliability study. 3 patients were excluded from a validity study due to the missing data. The following inclusion criteria were applied: patient's age over 18 years old and Polish nationality. Exclusion criteria were as follows: (i) age under 18 years old (ii) age over 60 years old and (iii) other lower limb injury. Invitation for participation in the study was performed during a phone call, where the goal as well as methodology of the study was presented. After that, patients were invited to the clinic, where after an examination by orthopaedic surgeon, the first test was performed. Patients received two questionnaires at the same time: ATRS-Polish and Polish version of EQ-5D-5L (previously adapted by Golicki [10]). The same test was performed after 5-10 days, then patients were asked during the telephone call to answer ATRS-Polish question one more time, to evaluate test-retest reliability. All patients gave written agreement for the participation in the study. This study was approved by the Bioethical Committee of the Regional Medical Council affiliated within Wielkopolska Izba Lekarska (opinion no. 191/2015, dated at 16.12.2015).

\section{Sample size}

There is no agreed optimum method for determining an appropriate sample size to evaluate aspects of validity for patient reported outcome measures [25]. Previous studies evaluating the aspects of validity and reliability of ATRS enrolled from 49 [5] to 112 patients [8]. We have reasoned that a case series of 71 patients would provide sufficient power to investigate the validity and reliability for the ATRS-Polish.

\section{Results}

\section{Translating process}

The procedure of translation and cultural adaptation of the original ATRS designed in Sweden into the Polish version 
Table 1 Demographic and clinical characteristics of participants

\begin{tabular}{ll}
\hline Characteristics & Number or mean \pm SD \\
\hline Total number of patients & 71 \\
ATRS test participants & 71 \\
ATRS re-test participants & 66 \\
Age (year) & $42.6( \pm 8)$ \\
Gender & \\
Male (\%) & $71(100 \%)$ \\
Female (\%) & $0(0 \%)$ \\
Involved side & \\
Right $(\%)$ & $30(42 \%)$ \\
Left $(\%)$ & $41(58 \%)$ \\
\hline
\end{tabular}

was implemented (Fig. 1). The final ATRS-Polish is presented in Supplementary Fig. 1. The original questions were easily translated, in most cases the English words were directly translated into Polish ones. Therefore, the translation did not involve significant changes. Moreover, as already observed during pre-testing experiments which involved ten patients with ankle pain and ten asymptomatic ones, filling of the ATRS-Polish form did not rise any difficulties when answering the questions. All patients involved in pre-testing considered the ATRS-Polish form as easy to follow, userfriendly and well adapted to activities of daily living. This initial feedback from the patients clearly indicated that the questions were clear and understandable. The patients usually required 3-5 min to complete the form. The score values obtained in the first and second experiment are presented in Table 2.

\section{Validity}

To validate the outcomes of newly constructed ATRS-Polish, the results were compared with already implemented EQ-5D-5L score in our clinic, previously adapted by Golicki et al. [10]. A strong correlation between the ATRS-Polish and a Polish EQ-5D-5L score was observed ( $r=-0.69$; $p<0.001$, Table 3). Additionally, Polish-ATRS was moderately correlated with the actual comfort parameter $(r=0.47$; $p<0.001)$.
Table 3 Validity as measured by correlation among questionnaires

\begin{tabular}{lcc}
\hline Measurement & $r$ & $p$ value \\
\hline ATRS vs EQ-5D-5L & -0.691 & $<0.000001$ \\
ATRS vs actual comfort & 0.474 & $<0.00001$ \\
\hline
\end{tabular}

Spearman's correlation coefficients $(r)$ for test-retest are presented

\section{Reliability}

The test-retest reliability of the ATRS-Polish was found to be excellent with the ICC value of $0.9(p<0.0001)$. Internal consistency of the ATRS-Polish was found to be excellent (Cronbach's alpha 0.93).

\section{Floor and ceiling effect}

There were no missing data for individual items of ATRS since all patients responded to all questions (response rate $100 \%)$. The ATRS scores were well distributed and ranged between 28.0 and 100.0 (Table 2). No patient was scored the lowest score in test or re-test. Maximum score was observed in $15 \%$ of patients in the first assessment and in $20 \%$ of patients in the second assessment. No floor or ceiling effects were observed.

\section{Discussion}

The most important finding of the present study was that the Polish version of the ATRS was reliable and valid and that the ATRS-Polish can be used in a Polish population to evaluate Achilles tendon rupture. Firstl, the translation of the original ATRS for a Polish context did not require major adaptations. All tested patients were able to correctly interpret and answer all activity-based questions included in the form. In this study, a combination of both, a paper-and-pencil questionnaire (during the first test) and a telephone survey (during the re-test) was applied. Such sampling appeared to be one of the strengths of the study, as almost $94 \%$ of patients enrolled in the first test decided to take part also in the second one. It could not be the case if a paper-and-pencil form for both tests (which would involve a second visit to the
Table 2 The absolute values of the ATRS-Polish and EQ-5D-5L scores

\begin{tabular}{lllllllll}
\hline & Min & First quartile & Median & Mean & Third quartile & Max & SD & SE \\
\hline ATRS test & 28.0 & 81.0 & 91.0 & 87.4 & 97.0 & 100 & 14.0 & 1.6 \\
ATRS re-test & 29.0 & 84.5 & 92.0 & 88.4 & 99.0 & 100 & 13.2 & 1.6 \\
Actual comfort & 50.0 & 80.0 & 90.0 & 87.6 & 95.0 & 100 & 9.3 & 1.1 \\
EQ-5D-5L & 5 & 5 & 6 & 6 & 7 & 13 & 1.3 & 0.2 \\
\hline
\end{tabular}

A mean value, median value, standard deviation (SD), standard error (SE), minimal and maximal values and first and third quartile of the outcomes are presented 
clinic) or an online survey (which could be highly dependent on the internet availability and/or the ability to proper use of the web tools) was enrolled. Moreover, a neutral setting of the responders during a telephone call in our study (in comparison to visit in a clinic which may influence the patients' responses) additionally minimized the environmental biases.

To gain more insight into the reliability of the ATRSPolish test, an extensive evaluation of the outcomes was performed. According to our results, the test was highly reproducible even when patients were asked to re-perform the test 10 days after initial study. The measured reliability was found to be excellent (ICC value of 0.90), which is in a good agreement with other studies based on translated versions of the original ATRS test: in Danish population (ICC $=0.91$, [9]), Italian (ICC $=0.96$, [26]), English $(\mathrm{ICC}=0.98,[5])$, Persian $(\mathrm{ICC}=0.98,[2])$ or Brazilian Portuguese $(\mathrm{ICC}=0.93,[27])$. By comparison, in the original ATRS study in Sweden [19], a significantly higher score was reported on the second test day compared with first test day when testing was performed twice within 2 weeks. The excellent test-re-test reliability indicated that the ATRS-Polish was stable over time and pointed into the possibility of detecting changes in patients with ATR. We can, therefore, reason that reproducibility of newly implemented ATRSPolish test is definitely a strong point of the present study.

None of the patients achieved the minimum ATRS score, and therefore, no floor effect was observed. This is comparable to the results from other studies, which vary from 0 to $1 \%[9,15] .15 \%$ of patients achieved the maximum score in ATRS-test and 20\% during the re-test, which is much more than the threshold observed in other studies $(0-14 \%)[9,14$, 15]. The absence of floor or ceiling effects in the current study reflected the reliability, content validity, and responsiveness of the ATRS-Polish.

No missing items were observed and that the questions were clear and were of value were indicated to the patient, since questions in questionnaires were often marked as "not applicable" by the patient.

However, when individual questions were considered, particular patients did not respond equally, especially to question no. 7 in test and re-test. This question concerned quick walking up the stairs or hills. One of the possible explanations to that issue might be that not all of the patients were capable of such activities, independently of their Achilles tendon injury. This issue has been already discussed by Ganestam et al., when they proposed that questions no. 7, 8 (concerning running) and 9 (involves jumping) were not well adapted to all patients [9].

The construct validity of the ATRS-Polish questionnaire was determined by comparing the ATRS with selected outcome measures. For that purpose, a validated Polish version of EQ-5D-5L test has been used as well as the actual comfort score. The validity analysis showed strong correlation between the ATRS-Polish and EQ-5D-5L score $(r=-0.69)$. Additionally, it showed moderate correlation between ATRS and actual comfort $(r=0.47)$. These validation findings are in a good accordance with other studies concerning crossnational ATRS adaptations [2, 5, 9, 14, 18, 21, 26, 27]. It has been commonly accepted that such moderate strong correlation is desirable [6,25], because quality of life EQ5D-5L score is not specifically designed for Achilles tendon ruptures [10].

The major limitation of this study is that only men were enrolled in the study. It is, therefore, impossible to conclude about gender influence on the ATRS-Polish reliability and validity.

ATRS-Polish is easy to apply, reliable, valid, consistent and comparable to the original English version. It may, therefore, possess a significant clinical relevance. Validity and reliability, sufficient to assess clinical outcomes in the Polish population has been demonstrated. Significant benefits to both, practice and research, include the use of ATRSPolish to identify patient's symptoms and limitations and to monitor patient's status over time. The therapeutic process can be enhanced by measuring the functional outcome and demonstrating the treatment effectiveness. Moreover, validated and reliable Polish version of an international ATRS test will definitely allow Polish clinicians to compare the results of their patients on a nationwide scale.

\section{Conclusions}

Summarizing, we propose for the first time a Polish version of the ATRS test. The possible use of the cross-culturally adapted ATRS-Polish may be considered to evaluate the clinical condition after Achilles tendon rupture in day-byday clinical practice in the Polish population. The implementation of ATRS-Polish test will enhance the whole therapeutic process of Achilles tendon rupture.

Author contributions PB and TP designed the study. PB, SR and MW-S enrolled patients. PB, SR, MW-S and MG performed literature search. PB wrote the initial draft. MG made statistical analysis. TP, SR, MW-S and MG provided comments and prepare the final version of the manuscript for publication. All authors read and approved the final manuscript.

\section{Compliance with ethical standards}

Conflict of interest All the authors declare that they have no conflict of interest related to the topic of this article.

Funding The study was financed from own (Rehasport Clinic in Poznan, Poland) resources.

Ethical approval All patients gave written agreement for the participation in the study. This study was approved by the Bioethical Com- 
mittee of the Regional Medical Council affiliated within Wielkopolska Izba Lekarska (opinion no. 191/2015, dated at 16.12.2015).

Open Access This article is distributed under the terms of the Creative Commons Attribution 4.0 International License (http://creativecommons.org/licenses/by/4.0/), which permits unrestricted use, distribution, and reproduction in any medium, provided you give appropriate credit to the original author(s) and the source, provide a link to the Creative Commons license, and indicate if changes were made.

\section{References}

1. Ahmad J, Repka M, Raikin SM (2013) Treatment of myotendinous Achilles ruptures. Foot Ankle Int 34:1074-1078

2. Ansari NN, Naghdi S, Hasanvand S et al (2016) Cross-cultural adaptation and validation of Persian Achilles tendon total rupture score. Knee Surg Sports Traumatol Arthrosc 24:1372-1380

3. Beaton DE, Bombardier C, Guillemin F, Ferraz (2000) Guidelines for the process of cross-cultural adaptation of self-report measures. Spine 25:3186-3191

4. Carmont MR, Silbernagel KG, Edge A, Mei-Dan O, Karlsson J, Maffulli N (2013) Functional outcome of percutaneous achilles repair: improvements in achilles tendon total rupture score during the first year. Orthop J Sports Med 1(1):2325967113494584

5. Carmont MR, Silbernagel KG, Nilsson-Helander K, Mei-Dan O, Karlsson J, Maffulli N (2013) Cross cultural adaptation of the achilles tendon total rupture score with reliability, validity and responsiveness evaluation. Knee Surg Sports Traumatol Arthrosc 21:1356-1360

6. Chan YH (2003) Biostatistics 104: correlational analysis. Singapore Med J 44:614-619

7. Chiodo CP, Glazebrook M, Bluman E, Cohen B, Femino J, Giza E (2010) AAOS clinical practice guideline summary. Diagnosis and treatment of acute achilles tendon rupture. J Am Acad Orthop Surg 18:503-510

8. Cui J, Jia Z, Zhi X et al (2017) The chinese version of achilles tendon total rupture score: cross-cultural adaptation, reliability and validity. Health Qual Life Outcomes 15(1):2

9. Ganestam A, Barfod K, Klit J, Troelsen A (2013) Validity and reliability of the achilles tendon total rupture score. J Foot Ankle Surg 52:736-739

10. Golicki D, Niewada M (2017) EQ-5D-5L Polish population norms. Arch Med Sci 13(1):191-200

11. Husted JA, Cook RJ, Farewell VT, Gladman DD (2000) Methods for assessing responsiveness: a critical review and recommendations. J Clin Epidemiol 53:459-468

12. Huttunen TT, Kannus P, Rolf C et al (2014) Acute achilles tendon ruptures: incidence of injury and surgery in Sweden between 2001 and 2012. Am J Sports Med 42:2419

13. Järvinen TA, Kannus P, Maffulli N, Khan KM (2005) Achilles tendon disorders: etiology and epidemiology. Foot Ankle Clin 10(2):255-266
14. Kaya Mutlu E, Celik D, Kiliçoglu Ö, Ozdincler AR, NilssonHelander K (2015) The Turkish version of the achilles tendon total rupture score: cross-cultural adaptation, reliability and validity. Knee Surg Sports Traumatol Arthrosc 23(8):2427-2432

15. Kearney RS, Achten J, Lamb SE, Parsons N, Costa ML (2012) The Achilles tendon total rupture score: a study of responsiveness, internal consistency and convergent validity on patients with acute achilles tendon ruptures. Health Qual Life Outcomes 10:24

16. Leppilahti J, Puranen J, Orava S (1996) Incidence of achilles tendon rupture. Acta Orthop Scand 67(3):277-279

17. Malagelada F, Clark C, Dega R (2016) Management of chronic achilles tendon ruptures-a review. Foot (Edinb) 28:54-60

18. Myhrvold SB, Sandnes $\varnothing$, Hoelsbrekken SE (2017) Validity and reliability of the Norwegian translation of the achilles tendon total rupture score. Knee Surg Sports Traumatol Arthrosc. doi:10.1007/ s00167-017-4689-1

19. Nilsson-Helander K, Thomeé R, Silbernagel KG, Thomeé P, Faxén E, Eriksson BI, Karlsson J (2007) The achilles tendon total rupture score (ATRS): development and validation. Am J Sports Med 35:421-442

20. O'Brien M (1992) Functional anatomy and physiology of tendons. Clin Sports Med 11(3):505-520

21. Opdam KT, Zwiers R, Wiegerinck JI, Kleipool AE, Haverlag R, Goslings JC, van Dijk CN (2016) Reliability and validation of the Dutch Achilles tendon Total Rupture Score. Knee Surg Sports Traumatol Arthrosc. doi:10.1007/s00167-016-4242-7

22. Piontek T, Bąkowski P, Ciemniewska-Gorzela K, Naczk J (2015) Novel technique of achilles tendon reconstruction using semitendinosus and gracilis tendon. Preliminary report. Ortop Traumatol Rehabil 17(6):619-625

23. Sheth U, Wasserstein D, Jenkinson R et al (2017) The epidemiology and trends in management of acute Achilles tendon ruptures in Ontario, Canada: a population-based study of 27607 patients. Bone Jt J 99-B:78-86

24. Suchak AA, Bostick G, Reid D et al (2005) The incidence of Achilles tendon ruptures in Edmonton, Canada. Foot Ankle Int 26:932-936

25. Terwee CB, Bot S, de Boer M, van der Windt DA, Knol DA, Dekker J, Bouter LM, de Vet HC (2007) Quality criteria were proposed for measurement properties of health status questionnaires. J Clin Epidemiol 60:34-42

26. Vascellari A, Spennacchio P, Combi A et al (2016) Cross-cultural adaptation and multi-centric validation of the Italian version of the achilles tendon total rupture score (ATRS). Knee Surg Sports Traumatol Arthrosc. doi:10.1007/s00167-016-4152-8

27. Zambelli R, Pinto RZ, Magalhães JM, Lopes FA, Castilho RS, Baumfeld D, Dos Santos TR, Maffulli N (2016) Development of the Brazilian Portuguese version of the achilles tendon total rupture score (ATRS BrP): a cross-cultural adaptation with reliability and construct validity evaluation. BMC Sports Sci Med Rehabil $8: 11$ 